\title{
On the Use of Minimum Volume Ellipsoids and Symplectic Capacities for Studying Classical Uncertainties for Joint Position-Momentum Measurements
}

\author{
Maurice A. de Gosson* \\ Universität Wien, NuHAG \\ Fakultät für Mathematik \\ A-1090 Wien
}

June 29, 2018

\begin{abstract}
We study the minimum volume ellipsoid estimator associates to a cloud of points in phase space. Using as a natural measure of uncertainty the symplectic capacity of the covariance ellipsoid we find that classical uncertainties obey relations similar to those found in nonstandard quantum mechanics.
\end{abstract}

Keywords: position-momentum measurements, minimum volume ellipsoid, symplectic capacity, uncertainty principle, Hamiltonian system

\section{Introduction}

Contrarily to what is often believed the Heisenberg uncertainty principle

$$
\Delta p_{j} \Delta x_{j} \geq \frac{1}{2} \hbar
$$

is not a statement about the accuracy of our measurement instruments; its derivation assumes on the contrary perfect instruments. The correct interpretation of Heisenberg's inequalities is the following (see e.g. Peres [15], p.93): if the same preparation procedure is repeated a large number of

${ }^{*}$ E-mail: maurice.de.gosson@univie.ac.at 
times, and is followed by either by a measurement of $x_{j}$, or by a measurement of $p_{j}$, the results obtained have standard deviations $\Delta x_{j}$ and $\Delta p_{j}$ satisfying (11). The same interpretation is of course true for the stronger RobertsonSchrödinger [17, 21] inequalities

$$
\left(\Delta p_{j}\right)^{2}\left(\Delta x_{j}\right)^{2} \geq \Delta\left(x_{j}, p_{j}\right)^{2}+\frac{1}{4} \hbar^{2}
$$

to which (11) reduce if one neglects the covariances $\Delta\left(x_{j}, p_{j}\right)^{2}$; they are complemented by the trivial inequalities

$$
\Delta p_{j} \Delta x_{k} \geq 0 \text { if } j \neq k, \Delta p_{j} \Delta p_{k} \geq 0, \Delta x_{j} \Delta x_{k} \geq 0
$$

which might be violated in a nonstandard form of quantum mechanics, noncommutative mechanics (NCQM, see Dias et al. 3] and the references therein), where the second and third inequalities (3) are be replaced with

$$
\Delta p_{j}^{2} \Delta p_{k}^{2} \geq \Delta\left(p_{j}, p_{k}\right)^{2}+\frac{1}{4} \theta_{j k}^{2}, \Delta x_{j}^{2} \Delta x_{k}^{2} \geq \Delta\left(x_{j}, x_{k}\right)^{2}+\frac{1}{4} \eta_{j k}^{2}
$$

where $\theta_{j k}^{2}=\theta_{k j}^{2}$ and $\eta_{j k}^{2}=\eta_{k j}^{2}$.

In classical statistical mechanics the situation is somewhat different: due to the inherent inaccuracy of the measurement apparatus there are uncertainties for all pairs of variables, conjugate or not. We are going to show in this article that despite the different nature of quantum and classical uncertainties, the latter can be described in a similar way, leading to inequalities which are formally identical to those of NCQM, namely

$$
\begin{aligned}
\Delta x_{j}^{2} \Delta x_{k}^{2} & \geq \Delta\left(x_{j}, x_{k}\right)^{2}+a_{j k}^{2} \\
\Delta p_{j}^{2} \Delta p_{k}^{2} & \geq \Delta\left(p_{j}, p_{k}\right)^{2}+c_{j k}^{2} \\
\Delta x_{j}^{2} \Delta p_{k}^{2} & \geq \Delta\left(x_{j}, p_{k}\right)^{2}+b_{j k}^{2}
\end{aligned}
$$

with $a_{j k}^{2}=a_{k j}^{2}, b_{j k}^{2}=b_{k j}^{2}, c_{j k}^{2}=c_{k j}^{2}$. We have actually already shown in a recent paper [5] (also see de Gosson and Luef [7]) that inequalities of the type (11) -(2) are by no means characteristic of quantum mechanics, and that there are formally similar statements in classical statistical mechanics; to sustain our claim we used tools from robust multivariate statistics (the Minimum Volume Ellipsoid method, reviewed in Section 2) together with a topological device, the notion of symplectic capacity, which we use as a natural device for measuring uncertainty. The symplectic capacity of a closed phase space set is the equatorial area of the largest ball that can be sent in this set using only symplectomorphisms (=canonical transformations). The symplectic capacity of the covariance ellipsoid is not related to its volume (it always has 
the dimension of an area), but rather to that of a classical action. We note that symplectic capacities have been used by Scheeres and his collaborators [11, 20] to study satellite guidance and constraints on spacecraft trajectories.

The aim of this paper is to extend the results in [5] and to show that these NCQM uncertainties also appear quite naturally in classical statistical mechanics.

Notation 1 We identify $\mathbb{R}^{n} \times \mathbb{R}^{n}$ with $\mathbb{R}^{2 n}$ and write $(x, p)=z$ if $x \in \mathbb{R}^{n}$ and $p \in \mathbb{R}^{n}$. We will view $x, p$ and $z$ as column vectors in all matrix computations. A symplectic form on $\mathbb{R}^{2 n}$ is a non-degenerate bilinear antisymmetric form on that space. Let $\alpha$ be a symplectic form on $\mathbb{R}^{2 n}$; the corresponding symplectic group is denoted $\operatorname{Sp}(2 n, \alpha)$ [it is the group of all linear automorphisms $S$ of $\mathbb{R}^{2 n}$ such that $\alpha\left(S z, S z^{\prime}\right)=\alpha\left(z, z^{\prime}\right)$ for all vectors $z, z^{\prime}$ in $\left.\mathbb{R}^{2 n}\right]$. We denote by $\sigma$ the standard symplectic form on $\mathbb{R}^{2 n}$ : $\sigma\left(z, z^{\prime}\right)=\left(z^{\prime}\right)^{T} J z$ if $z=(x, p), z^{\prime}=\left(x^{\prime}, p^{\prime}\right) ; J$ is the standard symplectic $\operatorname{matrix}\left(\begin{array}{cc}0_{n \times n} & I_{n \times n} \\ -I_{n \times n} & 0_{n \times n}\end{array}\right)$.

\section{The Minimum Volume Ellipsoid Method}

Let us consider a system of point-like particles; we assume that there are $n$ degrees of freedom and label the position coordinates and momenta $x=$ $\left(x_{1}, \ldots, x_{n}\right), p=\left(p_{1}, \ldots, p_{n}\right)$; we will also use the collective notation $z=(x, p)$. Assume now that we perform position and momentum measurements on a large number $K$ of identical copies of that system; we get a cloud $\mathcal{S}=$ $\left\{z_{1}, z_{2}, \ldots, z_{N}\right\}, N=n K$, of points in $\mathbb{R}^{2 n}$. An efficient method for studying that cloud consists in using the minimum volume ellipsoid (MVE) method for the multivariate location and scatter (Rousseeuw [18]). Geometrically speaking this method is an application of the John-Löwner theorem (see for instance Ball [2]): consider a subset $\left\{z_{i_{1}}, z_{i_{2}}, \ldots, z_{i_{k}}\right\}$ of $\mathcal{S}$; we will assume that the points $z_{i_{j}}$ are in general position, i.e. that they do not remain in some hyperplane of $\mathbb{R}^{2 n}$. This condition is sufficient and necessary for any ellipsoid containing these points to have positive volume. The points $z_{i_{1}}, z_{i_{2}}, \ldots, z_{i_{k}}$ determine a polyhedron in $\mathbb{R}^{2 n}$; we denote by $\mathcal{K}_{k}$ the convex hull of that polyhedron (it is the smallest subset of $\mathbb{R}^{2 n}$ containing $\left\{z_{i_{1}}, z_{i_{2}}, \ldots, z_{i_{k}}\right\}$ ). The John-Löwner theorem ensures us that there exists a unique ellipsoid $\mathcal{J}_{k}$ in $\mathbb{R}^{2 n}$ containing $\mathcal{K}_{k}$ and having minimum volume among all the ellipsoids having this property. Repeating this process for all subsets of the cloud $\mathcal{S}$ having $k$ elements in general position we get a family of ellipsoids; by definition the MVE is the one with the smallest volume. 
This method is practically implemented as follows (see e.g. Van Aelst and Rousseeuw [22], Hubert et al. [12]): choose an integer $k$ between $[N / 2]+$ 1 and $N([N / 2]$ the integer part of $N / 2)$; this constant $k$ determines the robustness of the resulting estimator; a common choice is

$$
k=\left[\frac{N+2 n+1}{2}\right] .
$$

By definition, the location and scatter estimators minimize the determinant of the matrices $M$ subject to the condition

$$
\#\left\{j:\left(z_{j}-\bar{z}\right)^{T} M^{-1}\left(z_{j}-\bar{z}\right) \leq m^{2}\right\} \geq k
$$

where the minimization is over all $\bar{z} \in \mathbb{R}^{2 n}$ and all positive definite symmetric matrices $M$ of size $2 n$. Here $m$ is a fixed constant, chosen so that the MVE estimator is a consistent estimator for of the covariance matrix for data coming from a multivariate normal distribution, that is

$$
m=\sqrt{\chi_{2 n, \alpha}^{2}}, \alpha=k / N
$$

where $\chi_{2 n, \alpha}^{2}$ is a chi-square distribution with $2 n$ degrees of freedom (see Lopuhäa and Rousseeuw [14]). One the pair $(M, \bar{z})$ is determined, the minimum volume ellipsoid (MVE) is the set of all $z$ in $\mathbb{R}^{2 n}$ such that

$$
(z-\bar{z})^{T} M^{-1}(z-\bar{z}) \leq m^{2} .
$$

The next step consists in associating to the MVE $\mathcal{J}$ a covariance matrix. For this one has to choose an adequate value $m_{0}$ for $m$; denoting the corresponding matrix $M$ by $\Sigma$ the MVE is the ellipsoid

$$
\mathcal{C}:(z-\bar{z})^{T} \Sigma^{-1}(z-\bar{z}) \leq m_{0}^{2}
$$

and $\Sigma$ is then precisely the covariance matrix.

We will write the covariance matrix in the form

$$
\Sigma=\left(\begin{array}{ll}
\Delta(x, x) & \Delta(x, p) \\
\Delta(p, x) & \Delta(p, p)
\end{array}\right)
$$

where $\Delta(x, x)=\left(\left(\Delta\left(x_{i}, x_{j}\right)\right)_{1 \leq i, j \leq n}\right.$ and so on. We will write, as is customary, $\Delta\left(x_{i}, x_{i}\right)=\Delta x_{i}^{2}$ and $\Delta\left(p_{i}, p_{i}\right)=\Delta p_{i}^{2}$; we have $\Delta\left(x_{i}, x_{j}\right)=\Delta\left(x_{j}, x_{i}\right)$, $\Delta\left(p_{i}, p_{j}\right)=\Delta\left(p_{j}, p_{i}\right), \Delta\left(x_{i}, p_{j}\right)=\Delta\left(p_{j}, x_{i}\right)$ hence the covariance matrix is symmetric. 


\section{A Condition on $\Sigma$}

Let $A=\left(a_{j k}\right)_{1 \leq j, k \leq n}$ and $C=\left(c_{j k}\right)_{1 \leq j, k \leq n}$ be two real antisymmetric matrices, and $B=\left(b_{j k}\right)_{1 \leq j, k \leq n}$ a real symmetric matrix. To $A, B, C$ we associate the $2 n \times 2 n$ antisymmetric matrix

$$
\Omega=\left(\begin{array}{cc}
A & B \\
-B & C
\end{array}\right)
$$

which is the most general form an antisymmetric of size $2 n$ can have. We will always assume that the matrix $\Omega$ is in addition invertible. This condition implies that the bilinear form $\omega$ on $\mathbb{R}^{2 n}$ defined by $\omega\left(z, z^{\prime}\right)=-\left(z^{\prime}\right)^{T} \Omega^{-1} z$ is a symplectic form on $\mathbb{R}^{2 n}$, and we denote by $\left(\mathbb{R}^{2 n}, \omega\right)$ the corresponding symplectic phase space. Notice that when $A=C=0$ and $B=I$ we have $\Omega=J=\left(\begin{array}{cc}0 & I \\ -I & 0\end{array}\right)$, the standard symplectic matrix. The general case can actually be reduced to the standard case:

Proposition 2 There exist linear automorphisms $F$ of $\mathbb{R}^{2 n}$ such that $\Omega=$ $F^{T} J F$; equivalently $F$ is a linear symplectomorphism $\left(\mathbb{R}^{2 n}, \sigma\right) \longrightarrow\left(\mathbb{R}^{2 n}, \omega\right)$, that is we have $\omega\left(F z, F z^{\prime}\right)=\sigma\left(z, z^{\prime}\right)$ for all $z$ and $z^{\prime}$.

Proof. Let $\mathcal{B}^{\sigma}=\left\{e_{1}^{\sigma}, \ldots, e_{n}^{\sigma}\right\} \cup\left\{f_{1}^{\sigma}, \ldots, f_{n}^{\sigma}\right\}$ and $\mathcal{B}^{\omega}=\left\{e_{1}^{\omega}, \ldots, e_{n}^{\omega}\right\} \cup\left\{f_{1}^{\omega}, \ldots, f_{n}^{\omega}\right\}$ be symplectic bases of $\left(\mathbb{R}^{2 n}, \sigma\right)$ and $\left(\mathbb{R}^{2 n}, \omega\right)$ respectively (i.e. $\omega\left(e_{j}^{\omega}, e_{k}^{\omega}\right)=$ $\omega\left(f_{j}^{\omega}, f_{k}^{\omega}\right)=0, \omega\left(f_{j}^{\omega}, e_{k}^{\omega}\right)=\delta_{j k}$ and similar relations for the $\sigma\left(e_{j}^{\sigma}, e_{k}^{\sigma}\right)$, etc. $)$. The automorphism $F$ defined by $F\left(e_{j}^{\sigma}\right)=e_{j}^{\omega}, F\left(f_{j}^{\sigma}\right)=f_{j}^{\omega}$ is a symplectomorphism $\left(\mathbb{R}^{2 n}, \sigma\right) \longrightarrow\left(\mathbb{R}^{2 n}, \omega\right)$.

We remark that this result (which can also be proved using the properties of the Pfaffian $P f(\Omega)$ ) is just a restatement of the linear (and global) version of Darboux's theorem [9] on the local equivalence of all symplectic manifolds with same dimension.

Let now $\Sigma$ be the covariance matrix as defined above, and consider the matrix

$$
\Sigma+i \Omega=\left(\begin{array}{cc}
\Delta(x, x)+i A & \Delta(x, p)+i B \\
\Delta(p, x)-i B & \Delta(p, p)+i C
\end{array}\right) .
$$

We observe that $\Sigma+i \Omega$ is Hermitian since $\Sigma$ is symmetric and $(i \Omega)^{*}=i \Omega$ since $\Omega$ is real antisymmetric. The eigenvalues of $\Sigma+i \Omega$ are thus real. From now on we will assume that these eigenvalue are nonnegative, that is $\Sigma+i \Omega$ is semi-definite positive, which we write

$$
\Sigma+i \Omega \geq 0
$$


(One can actually show that this condition automatically implies that $\Sigma>$ $0)$. Notice that we have in particular

$$
\Delta(x, x)+i A \geq 0, \quad \Delta(p, p)+i C \geq 0 .
$$

When $n=1$ the covariance matrix is just

$$
\Sigma=\left(\begin{array}{cc}
\Delta x^{2} & \Delta(x, p) \\
\Delta(p, x) & \Delta p^{2}
\end{array}\right)
$$

and the antisymmetric matrices $\Theta$ and $N$ are zero so that $\Omega=a J=$ $\left(\begin{array}{cc}0 & a \\ -a & 0\end{array}\right)$. The condition $\Sigma+i \Omega=\Sigma+i a J \geq 0$ is in this case equivalent to

$$
\left(\begin{array}{cc}
\Delta x^{2}+i a & \Delta(x, p) \\
\Delta(p, x) & \Delta p^{2}-i a
\end{array}\right) \geq 0
$$

which is in turn equivalent to the single inequality

$$
\Delta x^{2} \Delta p^{2} \geq \Delta(x, p)^{2}+a^{2} .
$$

This is of course formally the Robertson-Schrödinger inequality (2); in particular we have the Heisenberg-type inequality $\Delta x \Delta p \geq a$.

Let us extend the study to higher dimensions. When $n=2$ the matrices $A, B$, and $C$ are of the type

$$
A=\left(\begin{array}{cc}
0 & a \\
-a & 0
\end{array}\right), B=\left(\begin{array}{ll}
b & d \\
d & e
\end{array}\right), C=\left(\begin{array}{cc}
0 & c \\
-c & 0
\end{array}\right)
$$

so that $\Sigma+i \Omega$ is the $4 \times 4$ matrix

$$
\left(\begin{array}{cccc}
\Delta x_{1}^{2} & \Delta\left(x_{1}, x_{2}\right)+i a & \Delta\left(x_{1}, p_{1}\right)+i b & \Delta\left(x_{1}, p_{2}\right)+i d \\
\Delta\left(x_{2}, x_{1}\right)-i a & \Delta x_{2}^{2} & \Delta\left(x_{2}, p_{1}\right)+i d & \Delta\left(x_{2}, p_{2}\right)+i e \\
\Delta\left(p_{1}, x_{1}\right)-i b & \Delta\left(p_{1}, x_{2}\right)-i d & \Delta p_{1}^{2} & \Delta\left(p_{1}, p_{2}\right)+i c \\
\Delta\left(p_{2}, x_{1}\right)-i d & \Delta\left(p_{2}, x_{2}\right)-i e & \Delta\left(p_{2}, p_{1}\right)-i c & \Delta p_{2}^{2}
\end{array}\right)
$$

Recalling Sylvester's criterion [10, 13] which says that a Hermitian matrix is positive semidefinite if an only if all of its principal minors are nonnegative, the condition $\Sigma+i \Omega \geq 0$ implies that the principal minors of order two of 
$\Sigma+i \Omega$ must be $\geq 0$, we immediately get the inequalities

$$
\begin{aligned}
& \Delta x_{1}^{2} \Delta x_{2}^{2} \geq \Delta\left(x_{1}, x_{2}\right)^{2}+a^{2} \\
& \Delta x_{1}^{2} \Delta p_{1}^{2} \geq \Delta\left(x_{1}, p_{1}\right)^{2}+b^{2} \\
& \Delta p_{1}^{2} \Delta p_{2}^{2} \geq \Delta\left(p_{1}, p_{2}\right)^{2}+c^{2} \\
& \Delta x_{1}^{2} \Delta p_{2}^{2} \geq \Delta\left(x_{1}, p_{2}\right)^{2}+d^{2} \\
& \Delta x_{2}^{2} \Delta p_{1}^{2} \geq \Delta\left(x_{2}, p_{1}\right)^{2}+d^{2} \\
& \Delta x_{2}^{2} \Delta p_{2}^{2} \geq \Delta\left(x_{2}, p_{2}\right)^{2}+e^{2} .
\end{aligned}
$$

The same argument shows that more generally:

Proposition 3 Let $n \geq 2$. If the covariance matrix $\Sigma$ satisfies the condition $\Sigma+i \Omega \geq 0$ then the following uncertainty relations hold:

$$
\begin{aligned}
\Delta x_{j}^{2} \Delta x_{k}^{2} & \geq \Delta\left(x_{j}, x_{k}\right)^{2}+a_{j k}^{2} \\
\Delta p_{j}^{2} \Delta p_{k}^{2} & \geq \Delta\left(p_{j}, p_{k}\right)^{2}+c_{j k}^{2} \\
\Delta x_{j}^{2} \Delta p_{k}^{2} & \geq \Delta\left(x_{j}, p_{k}\right)^{2}+b_{j k}^{2} .
\end{aligned}
$$

In particular, if $\Omega=\varepsilon J, \varepsilon>0$, these conditions reduce to the RobertsonSchrödinger inequalities

$$
\Delta x_{j}^{2} \Delta p_{k}^{2} \geq \Delta\left(x_{j}, p_{k}\right)^{2}+\varepsilon^{2} .
$$

A warning: the group of inequalities (17)-(19) is not equivalent to the condition $\Sigma+i \Omega \geq 0$ as soon as $n>1$. Here is a simple counterexample in the case $n=2$ and $\Omega=J$ : choose $\eta=1$ and

$$
\Sigma=\left(\begin{array}{cccc}
1 & -1 & 0 & 0 \\
-1 & 1 & 0 & 0 \\
0 & 0 & 1 & 0 \\
0 & 0 & 0 & 1
\end{array}\right)
$$

This matrix is positive definite, and the inequalities above hold trivially (they reduce to equalities); the matrix $\Sigma+i J$ is however indefinite (its determinant is -1 ).

\section{The Symplectic Capacity of an Ellipsoid}

For the basics of symplectic geometry we are going to use we refer to Arnol'd [1, de Gosson [4] (Arnol'd uses the term "canonical transformation" for symplectomorphism; this terminology is usual in Physics). 
The condition $\Sigma+i \Omega \geq 0$ can be restated in terms of the symplectic capacity of the minimum volume ellipsoid $\mathcal{C}_{0}$ given by (11). Let us recall the definition of the notion of symplectic capacity (of which we have given a detailed discussion in de Gosson and Luef [7]). Let us call symplectic manifold a submanifold $U$ of $\mathbb{R}^{2 n}$ (possibly with boundary) equipped with a symplectic form $\alpha$. A symplectic capacity associates to every symplectic manifold $(U, \alpha)$ a number $c(U, \alpha) \geq 0$ or $+\infty$; this correspondence must satisfy the following axioms [9, 16]:

1. Monotonicity: If $\Phi:(U, \alpha) \longrightarrow(V, \beta)$ is a symplectic embedding, i.e. a diffeomorphism satisfying $\beta\left(\Phi(z), \Phi\left(z^{\prime}\right)=\alpha\left(z, z^{\prime}\right)\right.$ we must have $c(U, \alpha) \leq c(V, \beta)$

2. Conformality: For every real $\lambda \neq 0$ we have $c(U, \lambda \alpha)=\lambda^{2} c(U, \alpha)$;

3. Normalization: $c(B(R), \sigma)=c\left(Z_{j}(R), \sigma\right)=\pi R^{2}$; here $\sigma$ is the standard symplectic form, $B(R)$ the ball $|x|^{2}+|p|^{2} \leq R^{2}$ and $Z_{j}(R)$ the cylinder $x_{j}^{2}+p_{j}^{2} \leq R^{2}$.

In general symplectic capacities are not related to the notion of volume; this is already clear from the normalization condition $c\left(Z_{j}(R), \sigma\right)=\pi R^{2}$ which shows that as soon as $n>1$ the symplectic capacity of a region with infinite volume can be finite; in fact the conformality axiom shows that symplectic capacities behave as areas under dilations. Also note that symplectic capacities are extensive quantities, i.e. they do not depend directly on dimension, as volume does.

The monotonicity axiom implies that if there exists a symplectomorphism (=symplectic diffeomorphism) $\Phi:(U, \alpha) \longrightarrow(V, \beta)$ such that $\Phi(U)=$ $V$ then

$$
c(U, \alpha)=c(\Phi(U), \beta)=c(V, \beta)
$$

hence symplectic capacities are symplectic invariants. The basic example of a symplectic capacity is Gromov's width $11 c_{\mathrm{GR}}$. It is defined as follows: let $R_{\mathrm{GR}}$ be the "symplectic radius" of $U$ : it is the supremum of all radii of balls $B(R)$ that can be embedded in $U$ using symplectomorphisms of $\left(\mathbb{R}^{2 n}, \sigma\right)$. By definition the Gromov width of $U$ is then $c_{\mathrm{GR}}(U, \sigma)=\pi R_{\mathrm{GR}}^{2}$ (with $c_{\mathrm{GR}}(U, \sigma)=\infty$ if $R_{\mathrm{GR}}=\infty$ ). The fact that the normalization axiom is satisfied follows from Gromov's non-squeezing theorem [8], a deep property of symplectic topology which says that the ball $B(R)$ cannot be embedded inside a cylinder $Z_{j}(r)$ with radius $r<R$. If $U$ is a compact, connected

\footnotetext{
${ }^{1}$ Sometimes also called symplectic area.
} 
and simply connected domain in the symplectic plane $\left(\mathbb{R}^{2}, \sigma\right)=\left(\mathbb{R}^{2},-\right.$ det $)$ then $c_{\mathrm{GR}}(U, \sigma)$ is the area of $U$. There exist infinitely many symplectic capacities (and $c_{\mathrm{GR}}$ is the smallest of all) but they all agree on ellipsoids (see e.g. [9, 7]). We are going to give an explicit formula below, but let us first introduce the following notation and terminology. Let $M$ be a positive definite $2 n \times 2 n$ real matrix and consider the product $J M$. Its eigenvalues are those of the antisymmetric matrix $M^{1 / 2} J M^{1 / 2}$ and are thus of the type $\pm i \lambda_{\sigma, j}(M), j=1, \ldots, n$, with $\lambda_{\sigma, j}(M)>0$. The numbers $\lambda_{\sigma, j}(M)$ are called the $\sigma$ - eigenvalues of $M$; up to a simultaneous reordering of the variables $x_{j}$ and $p_{j}$ one can always assume that $\lambda_{\sigma, 1}(M) \geq \cdots \geq \lambda_{\sigma, n}(M)$. The ordered set

$$
\operatorname{Spec}_{\sigma}(M)=\left(\lambda_{\sigma, 1}(M), \ldots, \lambda_{\sigma, n}(M)\right)
$$

is then called the $\sigma$ - spectrum of $M$ (when it is understood that it is the standard symplectic structure which is used one speaks about symplectic eigenvalues and symplectic spectrum). One proves the following properties:

$$
M \leq M^{\prime} \Longrightarrow \lambda_{\sigma, j}(M) \leq \lambda_{\sigma, j}\left(M^{\prime}\right), j=1, \ldots, n
$$

where $M \leq M^{\prime}$ means that $M^{\prime}-M$ is semi-definite positive, and

$$
\left(\lambda_{\sigma, 1}\left(M^{-1}\right), \ldots, \lambda_{\sigma, n}\left(M^{-1}\right)\right)=\left(\lambda_{\sigma, n}(M)^{-1}, \ldots, \lambda_{\sigma, 1}(M)^{-1}\right)
$$

(see de Gosson [4], §8.3). Moreover there exists a symplectic matrix $S \in$ $\operatorname{Sp}(2 n, \sigma)$ such that

$$
S^{T} M S=\left(\begin{array}{cc}
\Lambda^{M} & 0 \\
0 & \Lambda^{M}
\end{array}\right)
$$

where $\Lambda^{M}$ is the diagonal matrix $\operatorname{diag}\left(\lambda_{\sigma, 1}(M) \geq \cdots \geq \lambda_{\sigma, n}(M)\right)$ (Williamson's diagonal form, see [1, 4, 9, 23]). Formula (23) implies the following: let $\mathcal{M}$ be the phase space ellipsoid by inequality $z^{T} M z \leq 1$. The the inverse image $S^{-1}(\mathcal{M})$ has the normal form

$$
\sum_{j=1}^{n} \lambda_{\sigma, j}(M)\left(x_{j}^{2}+p_{j}^{2}\right) \leq 1 .
$$

One can of course replace the matrix $J$ above by the antisymmetric nondegenerate matrix $\Omega=F^{T} J F$ ( $F$ is defined as in Proposition 2). Considering as above the symplectic form $\omega\left(z, z^{\prime}\right)=-\left(z^{\prime}\right)^{T} \Omega^{-1} z$ the $\omega$-spectrum of $M$ is the decreasing sequence

$$
\operatorname{Spec}_{\omega}(M)=\left(\lambda_{\omega, 1}(M), \ldots, \lambda_{\omega, n}(M)\right)
$$


of positive numbers such that the $\pm i \lambda_{\omega, j}(M)\left(\lambda_{\omega, j}(M)>0\right)$ are the eigenvalues of of $\Omega M$; the properties (21) and (22) hold mutatis mutandis, replacing the subscript $\sigma$ with $\omega$. We have:

$$
\operatorname{Spec}_{\omega}(M)=\operatorname{Spec}_{\sigma}\left(F M F^{T}\right) .
$$

To prove this it is sufficient to show that $\Omega M$ and $J\left(F M F^{T}\right)$ have the same eigenvalues. Suppose $\Omega M=F^{T} J F M z=\lambda z$ for some complex number $\lambda$ and $z \neq 0$. This is equivalent to $J F M z=\lambda\left(F^{T}\right)^{-1} z$ and hence to $J F M F^{T}\left[\left(F^{T}\right)^{-1} z\right]=\lambda\left[\left(F^{T}\right)^{-1} z\right]$ which proves our claim.

Proposition 4 Let $M$ be definite positive and consider the ellipsoids $\mathcal{M}^{+}=$ $\left\{z: z^{T} M z \leq 1\right\}$ and $\mathcal{M}^{-}=\left\{z: z^{T} M^{-1} z \leq 1\right\}$ in $\mathbb{R}^{2 n}$. For every symplectic capacity c we have

$$
c\left(\mathcal{M}^{+}, \sigma\right)=\pi / \lambda_{\sigma, 1}(M) \quad, \quad c\left(\mathcal{M}^{-}, \sigma\right)=4 \pi \lambda_{\sigma, n}(M) .
$$

Proof. See for instance [7, 9] for a proof of the first formula (26). The second formula follows from the first in view of the equality (22).

The first formula (26) shows that the symplectic capacity of $\mathcal{M}$ is the area of the intersection of that ellipsoid with the $x_{1}, p_{1}$ plane once it has been put in normal form (24). This again shows that the symplectic capacity is related to an area, and not to volume. In fact, using the invariance of the action form one can restate the first formula (26) in the following way:

$$
c(\mathcal{M}, \sigma)=\oint_{\gamma_{\min }} p d x=\frac{1}{2} \oint_{\gamma_{\min }} p d x-x d p
$$

where $\gamma$ is the shortest Hamiltonian orbit carried by the surface of the ellipsoid $\mathcal{M}$ (see de Gosson and Luef [7]); the symplectic capacity of an ellipsoid is thus explicitly expressed in terms of a dynamical action.

\section{Application to the Uncertainty Principle}

Let us now prove the main result of this article. Recall (formula (11)) that the covariance matrix is the set

$$
\mathcal{C}=\left\{z:(z-\bar{z})^{T} \Sigma^{-1}(z-\bar{z}) \leq m_{0}^{2}\right\}
$$

for some suitable choice of the real number $m_{0}$. 
Theorem 5 The condition $\Sigma+i \Omega \geq 0$ for the covariance matrix $\Sigma$ is equivalent (for every symplectic capacity c) to the condition

$$
c(\mathcal{C}, \omega)=c\left(\left(F^{T}\right)^{-1} \mathcal{C}, \sigma\right) \geq \pi m_{0}^{2} \lambda_{n}(\Sigma)
$$

where $\lambda_{n}(\Sigma)$ is the largest $\sigma$ - eigenvalue of $\Sigma$. If (29) is satisfied then we have

$$
\begin{aligned}
\Delta x_{j}^{2} \Delta x_{k}^{2} & \geq \Delta\left(x_{j}, x_{k}\right)^{2}+a_{j k}^{2} \\
\Delta p_{j}^{2} \Delta p_{k}^{2} & \geq \Delta\left(p_{j}, p_{k}\right)^{2}+c_{j k}^{2} \\
\Delta x_{j}^{2} \Delta p_{k}^{2} & \geq \Delta\left(x_{j}, p_{k}\right)^{2}+b_{j k}^{2} .
\end{aligned}
$$

Proof. Since translations are symplectomorphisms in any symplectic structure, the ellipsoid $\mathcal{C}$ has the same symplectic capacity as the centered ellipsoid $z^{T} \Sigma^{-1} z \leq m_{0}^{2}$. The result now follows from Proposition 4 above with $M=m_{0}^{-1} \Sigma^{-1}$.

\section{Discussion}

The notion of symplectic capacity appears as a device allowing to measure in a geometrical way the size of the MVE in a new way. The inequalities

$$
\begin{aligned}
\Delta x_{j}^{2} \Delta x_{k}^{2} & \geq \Delta\left(x_{j}, x_{k}\right)^{2}+a_{j k}^{2} \\
\Delta p_{j}^{2} \Delta p_{k}^{2} & \geq \Delta\left(p_{j}, p_{k}\right)^{2}+c_{j k}^{2} \\
\Delta x_{j}^{2} \Delta p_{k}^{2} & \geq \Delta\left(x_{j}, p_{k}\right)^{2}+b_{j k}^{2} .
\end{aligned}
$$

are not equivalent to the condition $c(\mathcal{C}, \omega) \geq \pi m_{0}^{2} \lambda_{n}^{\Sigma}$ but are implied by it. Therefore, $c(\mathcal{C}, \omega) \geq \pi m_{0}^{2} \lambda_{n}(\Sigma)$ can be viewed as a stronger -but naturalversion of the uncertainty principle. Its usefulness might very well come from the fact that the condition $c(\mathcal{C}, \omega) \geq \pi m_{0}^{2} \lambda_{n}(\Sigma)$ is invariant under arbitrary symplectic transformations (linear or not). It is in particular preserved under Hamiltonian time evolution, since Hamiltonian flows consist of symplectomorphisms.

Acknowledgement 6 This work has been financed by the Austrian Research Agency FWF (Projektnummer P20442-N13). 


\section{References}

[1] Arnol'd V I, 1989 Mathematical Methods of Classical Mechanics, Graduate Texts in Mathematics, 2nd edition, Springer-Verlag

[2] Ball K M, 1992 Ellipsoids of maximal volume in convex bodies, Geom. Dedicata 41(2), 241-250

[3] Dias, N C, de Gosson, M, Luef, F, and Prata, J N, 2010, A deformation quantization theory for noncommutative quantum mechanics, J. Math. Phys. 51072101

[4] de Gosson M, 2006 Symplectic Geometry and Quantum Mechanics, Birkhäuser, Basel, series "Operator Theory: Advances and Applications" (subseries: "Advances in Partial Differential Equations"), Vol. 166

[5] de Gosson M, 2009 The Symplectic Camel and the Uncertainty Principle: The Tip of an Iceberg?, Found. Phys. 99 194-214

[6] de Gosson M and Luef F, 2007 Quantum States and Hardy's Formulation of the Uncertainty Principle: a Symplectic Approach, Lett. Math. Phys. 80 69-82

[7] de Gosson M and Luef F, 2009 Symplectic Capacities and the Geometry of Uncertainty: the Irruption of Symplectic Topology in Classical and Quantum Mechanics, Physics Reports 484 131-179

[8] Gromov M, 1985 Pseudoholomorphic curves in symplectic manifolds, Inventiones Mathematica 82 307-347

[9] Hofer H and Zehnder E, 1994 Symplectic Invariants and Hamiltonian Dynamics, Birkhäuser Advanced texts (Basler Lehrbücher), Birkhäuser Verlag

[10] Horn R A and Johnson C R, 1985 Matrix analysis, Cambridge University Press

[11] Hsiao F.-Y and Scheeres D J, 2007 Fundamental Constraints on Uncertainty Relations in Hamiltonian Systems, IEEE Transactions In Automatic Control 52(4) 686-691

[12] Hubert M, Rousseeuw, P J, and Van Aelst S, 2008 High-Breakdown Robust Multivariate Methods, Statistical Science 23(1) 92-119 
[13] Johnson R C (ed.), 1990 Matrix Theory and Applications, Amer. Math. Soc., $R I$

[14] Lopuhäa H P and Rousseeuw P J, 1991 Breakdown points of affine equivariant estimators of multivariate location and covariance matrices, The Annals of Statistics 19(1) 229-248

[15] Peres A, 1993 Quantum Theory: Concepts and Methods, Kluwer Academic Publishers

[16] Polterovich L, 2001 The Geometry of the Group of Symplectic Diffeomorphisms, Lectures in Mathematics, Birkhäuser

[17] Robertson H P, 1929 The uncertainty principle, Phys. Rev. 34 163-164

[18] Rousseeuw P J, 1985 Multivariate estimation with high breakdown point. In Grossmann, W, Pflug, G, Vicenze, I \& Wertz, (Eds.), Mathematical Statistics and Applications, Vol. B., Riedel Publishing, Dordrecht the Netherlands 283-297

[19] Rousseeuw P J and Leroy A M, 1987 Robust regression and outlier detection, John Wiley \& Sons, New York

[20] Scheeres D J, Hsiao F.-Y, Park R S, Villac B F, and Maruskin J M, 2006 Fundamental Limits on Spacecraft Orbit Uncertainty and Distribution Propagation, Journal of the Astronautical Sciences 54 505-523

[21] Schrödinger E, 1930 Zum Heisenbergschen Unschärfeprinzip, Berliner Berichte 296-303 [English translation: Angelow, A., Batoni, M.C.: About Heisenberg Uncertainty Relation. Bulg. Journal of Physics, 26, nos.5/6, 193-203 (1999), and http://arxiv.org/abs/quant-ph/9903100

[22] Van Aelst S and Rousseeuw P J, 2009 Minimum volume ellipsoid, Wiley Interdisciplinary Reviews: Computational Statistics 1(1) 71-82

[23] J. Williamson, 1936 On the algebraic problem concerning the normal forms of linear dynamical systems, Amer. J. of Math. 58 141-163. 